\title{
VolP codec selection for digital PLC systems
}

\section{Maryam Shabro, Behnam Gholamrezazadeh Family, Vahid Hammiaty Vaghef}

Communication Department, Niroo Research Institute, Tehran, Iran

Email address:

mshabro@nri.ac.ir (M. Shabro), behnam_gh_f@yahoo.com (B. G. Family), vvaghef@nri.ac.ir (V. H. Vaghef)

\section{To cite this article:}

Maryam Shabro, Behnam Gholamrezazadeh Family, Vahid Hammiaty Vaghef. VoIP Codec Selection for Digital PLC Systems. Journal of Electrical and Electronic Engineering. Special Issue: Research and Practices in Electrical and Electronic Engineering in Developing Countries. Vol. 3, No. 2-1, 2015, pp. 46-49. doi: 10.11648/j.jeee.s.2015030201.20

\begin{abstract}
IP-based networks developments highly integrate the communication systems and significantly reduce the installation cost. Digital PLC systems with the capability of Ethernet and VoIP services are designed because of the demand of new communication services in the electric power industry. Also due to the limited bandwidth in digital PLC systems, there are several techniques for optimal use of transmission capacity. In this paper, the possibility of using VoIP services in digital PLC systems has been investigated. Many conventional standards are studied for several parameters such as total algorithm delay, voice payload size/duration, packets per second, and bandwidth to select the best codec. Finally, a proper codec is recommended to employ in the digital PLC's VoIP service according to the two important factors in quality of voice services, i.e. available bandwidth and latency.
\end{abstract}

Keywords: Voice codec, Bandwidth, Delay, Digital PLC, PLC Link Transition, VoIP

\section{Introduction}

Power Line Carrier (PLC) technology has been widely used by electrical power utilities for telecommunication purposes. It utilizes high voltage transmission lines as a communication media. Reputable companies have presented Digital PLC (DPLC) systems by using digital modulation technology, audio compression and Time Division Multiplexing (TDM) in order to increase the transmission capacity of traditional analog PLCs. Therefore, the DPLC's can provide more voice and data services. New DPLC systems have provided data transmission rates between $20-200 \mathrm{kbps}$ at $4 \mathrm{kHz}$ to $32 \mathrm{kHz}$ bandwidth by using compression and signal processing technologies. With higher communication rates by using DPLC systems, it is possible to provide Ethernet data transmission between two substations; in this case PLC acts as an Ethernet bridge [1]. Some applications of Ethernet services in the power utilities are:

- Connecting RTU to SCADA centre or TCP-IP-based network.

- LAN interconnection for extracting data from the HV substation.

- Providing VoIP services.

- Providing E-mail and Internet services.

Ethernet bridging over DPLC is a reliable and economical solution for providing a low throughput Ethernet access for specific operational applications in a HV substation without fiber or microwave coverage. As for other low capacity bridging technologies, it is important to optimize the payload and therefore the size of the Ethernet frames when the Ethernet system includes a DPLC bridge.

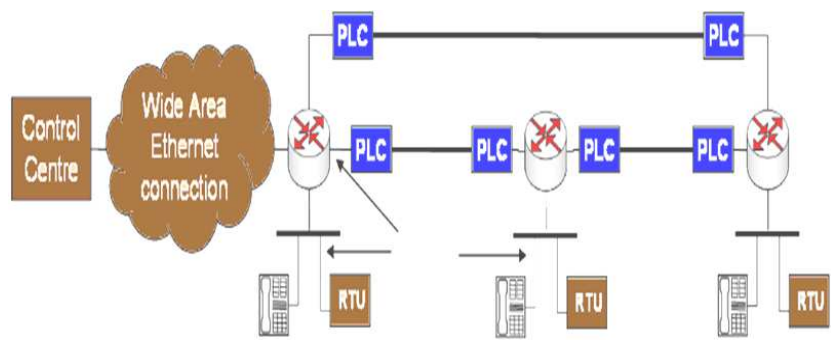

Figure 1. Typical Ethernet bridging over DPLC.

In this article, the reliable capacity of DPLCs under real condition, the framing in VoIP services, bandwidth reduction techniques for VoIP services, the possibility of using VoIP services in DPLCs and comparison of various codec regard to their bandwidth requirements are discussed. Also effective factors in quality of VoIP communication in telecommunication networks using DPLCs to choose suitable codec for the VoIP services through DPLC has been examined. 


\section{Available Bit Rates in Reliable Communication with DPLC System}

PLC performance and bandwidth capabilities depend upon the transmission line conditions. The PLC link's maximum transmission rate is determined by the available frequency bandwidth, which depends upon the density of the frequency plan and hence the number of PLC links in the immediate vicinity. The available transmission rate depends also upon the channel's Signal to Noise Ratio (SNR) and consequently upon the line noise and attenuation. The noise across HV PLC links is mainly due to the corona effect (ionization of the air) depending upon atmospheric conditions (air humidity, rain, pollution, and ice over the transmission line). According to Fig. 2, typically, with an SNR of about $35 \mathrm{~dB}$, the system can operate at $8 \mathrm{bps} / \mathrm{Hz}$ (32 kbps in $4 \mathrm{kHz}$ ) [2]. In order to assure high availability of bandwidth, the performance of the PLC link must be estimated under adverse atmospheric conditions. So, with an SNR of about $25 \mathrm{~dB}$, the system can operate at $6 \mathrm{bps} / \mathrm{Hz}(24 \mathrm{kbps}$ in $4 \mathrm{kHz})$.

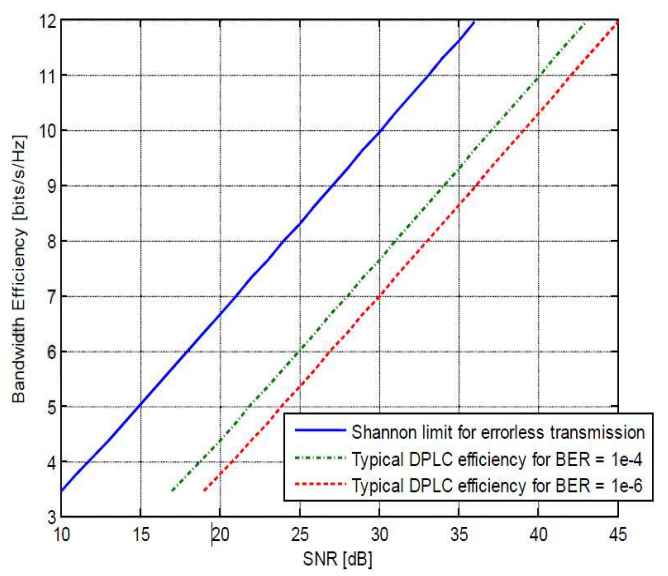

Figure 2. Typical DPLC bandwidth efficiency.

Clearly, these are the gross data rate. The specific value of this Gross Bit Rate depends on the implementation. In DPLC this overhead is due to the synchronization and TDM framing. In addition, there are some services which have higher priority to access the channel (such as dispatching voice channels and data from SCADA systems). Therefore, a part of the bit rate is used for these services (typically around 10kbps).

\section{VoIP Communication and Framing}

In a VoIP service as shown in Fig. 3, the following steps is necessary to generate and use of voice data packets:

- Sampling analog signals and converting them to digital signals by a codec;

- Data compression for optimal use of bandwidth;

- Using a real-time protocol (RTP) to control the sequence of received voice packets;

- A signaling protocol used to invoke user (H.323, SIP);

- Extract audio from received data packet;

- Decompressing received audio information and converting digital data to analog signal by a soundcard or phone.

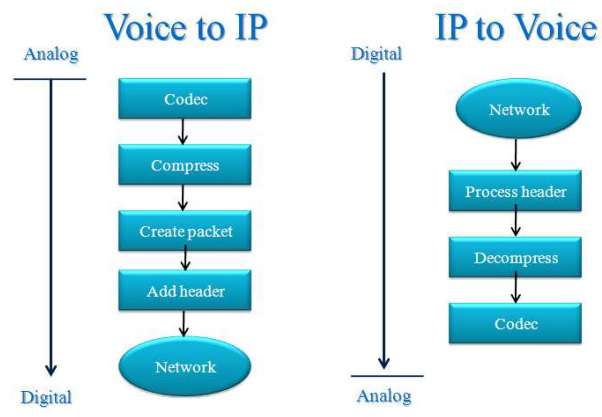

Figure 3 . Blocks in VoIP Services

Also the RSVP (Resource Reservation Protocol) is used in a VoIP connection. This protocol is responsible for service quality management function. RSVP is a QoS protocol for managing bandwidth allocation for Internet media connections (e.g. a VoIP voice connection). This protocol uses network resources and reserves bandwidth for voice transmission.

In general, VoIP packets consist of two parts, the voice payload and header (Fig. 4). As mentioned before, after converting the analog voice signal to digital data, to optimize the use of available bandwidth, the digital data should be compressed based on a suitable algorithm. The ITU Institute has developed numerous standards in this field. The type of codec determines audio sampling rate and actual required bandwidth. VoIP's overhead includes three protocols IP, UDP and RTP.

The RTP identifies the samples sequence (Fig. 5). The RTP also provides a method for diagnosing delay and Jitter [3]. The UDP specifies packages destination port. This protocol does not have any information about the packet sequence and does not provide any guarantee of delivery. The IP delivers the packets to the host. This protocol like UDP protocol does not guarantee delivering of all packets or delivering them in the same order they were sent.

The IP, UDP, and RTP header add a total of 40 bytes per packet. Besides, the MAC layer has about 42 bytes. So the most amount of communication bandwidth consists of these overheads. Besides the bandwidth required for VoIP depends on the type of network and use of Silence Suppression or Voice Activity Detection (VAD) techniques.

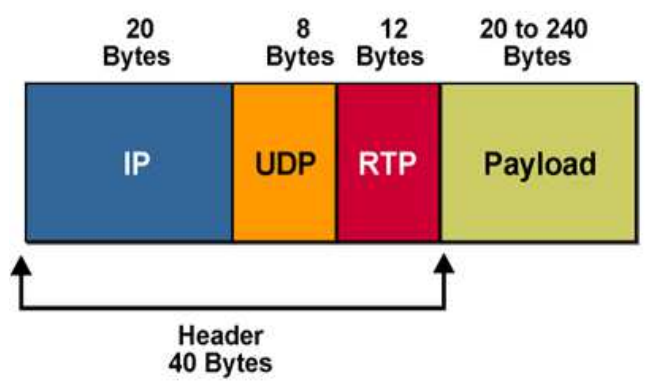

Figure 4. Structure of VoIP packets. 


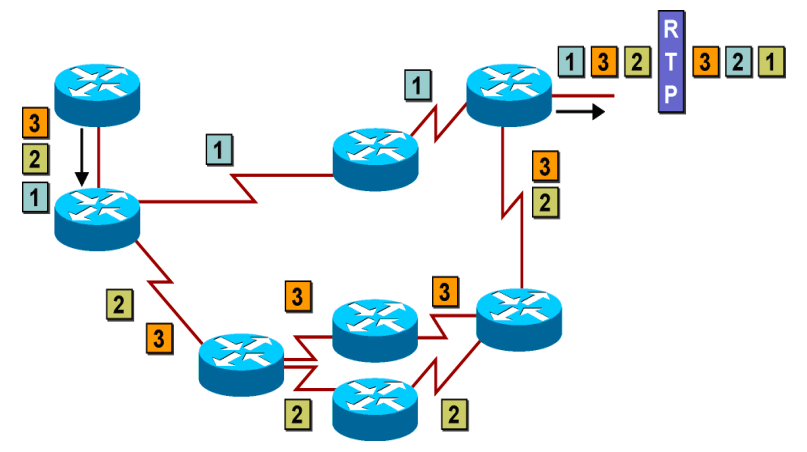

Figure 5. RTP's role in sorting packets.

\section{Reducing Required Bandwidth for a VoIP Communication over PLC Networks}

Due to the limited capacity of DPLC systems to provide IP based services, considerations for improving bandwidth efficiency will be essential. In this section some methods for reducing VoIP packet's size and the possibility of using in DPLC systems is investigated.

- In a Point-to-point communications it is possible to decrease 40 bytes of overhead to 2 or 4 bytes with a series of compression methods on overheads of IP, UDP, and RTP. DPLC systems use the point-to-point links, thus this compression method can be applied.

- In DPLC systems according to the TDM method for multiplexing different data services, some overhead will be added to the data frame. Therefore there is no need to send the MAC layer overheads (42 bytes). For example if HDLC framing is used at least 4 bytes as overhead and CRC is added to the contents of each packet which is much less than the MAC layer overheads.

- Few frames of audio data can be sent in one packet with the same overhead. It should be noted that prolonged packet increases transmission delay of sound. Moreover, regard to the behavior of noise on PLC network, a packet loss, will cause to lose all the frames and the quality of sound service will drop significantly.

Selecting the appropriate voice codec is important. If high compression rate codec is used to reduce bandwidth requirements, the complexity of algorithm and communication delay will be increase.

For example, take the codec G.729 (8 kbps), the minimum bandwidth required for VoIP communication without the compression of header is $31.2 \mathrm{kbps}$ and with the compression of header is $11.6 \mathrm{kbps}$ and the delay caused by algorithm is $25 \mathrm{~ms}$, also for the codec G.723.1 (5.3 kbps), the minimum bandwidth required for VoIP connection without the compression of header is $20.8 \mathrm{kbps}$ and with the compression is $7.7 \mathrm{kbps}$ and the delay caused by algorithm is $37.5 \mathrm{~ms}$. As described in Part 2 of this article, due to the limited capacity of DPLC systems and required bandwidth by codec for VoIP services, only the standard codec G.723.1 (5.3 kbps), G.723.1 (6.3 kbps)[4], and the G.729 (8 kbps)[5] are suitable for VoIP connection using DPLCs. The Comparison of some voice codecs are listed in Table 1.

Table 1. Comparison of some voice codecs.

\begin{tabular}{|c|c|c|c|c|c|c|c|c|}
\hline \multicolumn{4}{|l|}{ Codec Information } & \multicolumn{5}{|c|}{ Bandwidth Calculations } \\
\hline $\begin{array}{l}\text { Codec \& Bit Rate } \\
\text { (kbps) }\end{array}$ & $\begin{array}{l}\text { Codec } \\
\text { Sample } \\
\text { Size } \\
\end{array}$ & $\begin{array}{l}\text { Codec } \\
\text { Sample } \\
\text { Interval }\end{array}$ & $\begin{array}{l}\text { Total } \\
\text { algorithm } \\
\text { delay }\end{array}$ & $\begin{array}{l}\text { Voice } \\
\text { Payload } \\
\text { Size } \\
\end{array}$ & $\begin{array}{l}\text { Voice } \\
\text { Payload } \\
\text { Duration }\end{array}$ & $\begin{array}{l}\text { Packets Per } \\
\text { Second (PPS) }\end{array}$ & $\begin{array}{l}\text { Bandwidth } \\
\text { w/cRTP }\end{array}$ & $\begin{array}{l}\text { Ethernet } \\
\text { Bandwidth }\end{array}$ \\
\hline G.711 (64 kbps) & 80 Bytes & $10 \mathrm{~ms}$ & $20 \mathrm{~ms}$ & 160 Bytes & $20 \mathrm{~ms}$ & 50 & $67.6 \mathrm{kbps}$ & $87.2 \mathrm{kbps}$ \\
\hline G.723.1(6.3 kbps) & 24 Bytes & $30 \mathrm{~ms}$ & $37.5 \mathrm{~ms}$ & 24 Bytes & $30 \mathrm{~ms}$ & 33.3 & $8.8 \mathrm{kbps}$ & $21.9 \mathrm{kbps}$ \\
\hline G.723.1(5.3 kbps $)$ & 20 Bytes & $30 \mathrm{~ms}$ & $37.5 \mathrm{~ms}$ & 20 Bytes & $30 \mathrm{~ms}$ & 33.3 & $7.7 \mathrm{kbps}$ & $20.8 \mathrm{kbps}$ \\
\hline G.726 (32 kbps) & 20 Bytes & $5 \mathrm{~ms}$ & $20 \mathrm{~ms}$ & 80 Bytes & $20 \mathrm{~ms}$ & 50 & $35.6 \mathrm{kbps}$ & $55.2 \mathrm{kbps}$ \\
\hline G.726 (24 kbps) & 15 Bytes & $5 \mathrm{~ms}$ & $20 \mathrm{~ms}$ & 60 Bytes & $20 \mathrm{~ms}$ & 50 & $27.6 \mathrm{kbps}$ & $47.2 \mathrm{kbps}$ \\
\hline G.728 (16 kbps) & 10 Bytes & $5 \mathrm{~ms}$ & $20 \mathrm{~ms}$ & 60 Bytes & $30 \mathrm{~ms}$ & 33.3 & $18.4 \mathrm{kbps}$ & $31.5 \mathrm{kbps}$ \\
\hline G.729 (8 kbps) & 10 Bytes & $10 \mathrm{~ms}$ & $25 \mathrm{~ms}$ & 20 Bytes & $20 \mathrm{~ms}$ & 50 & $11.6 \mathrm{kbps}$ & $31.2 \mathrm{kbps}$ \\
\hline
\end{tabular}

\section{Effective Parameters on a VoIP Quality over PLC Networks}

The quality of VoIP connection depends on parameters such as delay, jitter and echo. Telephony services due to the Hybrid sections are faced with echo problem. If the communication delay is increased, echo will be felt more and this will have a considerable decline in the quality of service. ITU-T G.131 standard has described the acceptable echo versus delay [6]. The standard methods for echo cancellation (such as ITU-T G.168) will solve the echo problems in a channel with limited delay. The talker overlap problem will encounter with delay more than $250 \mathrm{~ms}$ which diminishes the quality of service.
UDP protocol is used in a VoIP connection so it is not possible to ask for a lost data packet. According to ITU-T standard if the network packet loss rate is $10 \%$ or more, the network is unreliable. A Service quality control procedure (RSVP) is used to solve this problem for the VoIP services but it is not possible to use RSVP for channel reservation with the DPLC system, because there are other services (RTU's data, DTS phone, etc) that have higher priority than VoIP. The whole end-to-end delay is an important factor that affects the quality of service. According to ITU-T G.114 standard end-to-end delay less than $150 \mathrm{~ms}$ is considered as a clear channel. The end-to-end delay over $400 \mathrm{~ms}$ designs is not acceptable for most applications [7].

The most important factors that cause a delay in VoIP 
communication are:

Coder delay: Also called processing delay, coder delay is the time taken by the DSP to compress a block of samples.

Packetization delay: Packetization delay is the time it takes to fill a packet payload with encoded or compressed speech.

Queuing delay: Queuing delay is a variable delay and is dependent on the trunk speed and the state of the queue.

Serialization delay: Serialization delay is the fixed delay that is required to clock a voice or data frame onto the network interface.

Network delay: The delay of network equipments that interconnects the endpoint locations is the source of the longest voice-connection delays. These delays are also the most difficult to quantify.

De-jitter buffer delay: Because speech is a constant bit-rate service, the jitter from all the variable delays must be removed before the signal leaves the network. The de-jitter buffer transforms the variable delay into a fixed delay by holding the first sample that is received for a period of time before playing it out. This holding period is known as the initial play out delay [8].

Table 2. Delay of VoIP service with DPLC for two type of codec.

\begin{tabular}{lll}
\hline G.729 & G.723.1 & Codec type \\
\hline $35 \mathrm{~ms}$ & $97 \mathrm{~ms}$ & Coder delay \\
$25 \mathrm{~ms}$ & $37.5 \mathrm{~ms}$ & Packetization delay \\
$30 \mathrm{~ms}$ & $30 \mathrm{~ms}$ & Queuing delay \\
$50 \mathrm{~ms}$ & $50 \mathrm{~ms}$ & Network delay \\
$40-60 \mathrm{~ms}$ & $60-90 \mathrm{~ms}$ & De-jitter buffer delay \\
$180-200 \mathrm{~ms}$ & $274.5-304.5 \mathrm{~ms}$ & Total Communication delay \\
\hline
\end{tabular}

For instance, Table 2 shows delay for G.723.1 and G.729 codec to use VoIP service with DPLC system.

With increasing number of nodes, the transmission delay of each node should be considered.

Table 3. Delay of VoIP connections based on the number of DPLC links in a network.

\begin{tabular}{lll}
\hline G.729 Delay & G.723.1 Delay & Link connection \\
\hline $180-200 \mathrm{~ms}$ & $274-304 \mathrm{~ms}$ & Point-to-Point \\
$260-280 \mathrm{~ms}$ & $354-384 \mathrm{~ms}$ & One transition node \\
$340-360 \mathrm{~ms}$ & $434-464 \mathrm{~ms}$ & Two transition nodes \\
$420-440 \mathrm{~ms}$ & $514-544 \mathrm{~ms}$ & Three transition nodes \\
\hline
\end{tabular}

Table 3 shows the delay between the two types of VoIP with G.723.1 and G.729 codec based on the number of links contained in DPLC communication. As noted above, based on the ITU-T G.114 standard the end-to-end delay, over $400 \mathrm{~ms}$ are not acceptable for most applications. So the audio codec based on G.723.1 standard is suitable for point to point and with one node between DPLCs and the audio codec based on G.729 standard is suitable for a maximum of two node per communication link.

\section{Discussion and Conclusions}

Available bit rates in DPLC systems due to the limited bandwidth, limited free frequency channel and noise restrictions is far from the theory. Therefore, compression methods for VoIP services in DPLC systems should be employed for optimal use of the channel capacity. Different compression algorithms can increase the delay and degrade the quality of service. Based on the available rates in DPLC and acceptable delay in a telephone communication services, the G.723.1 and G.729 codecs are recommended in this paper as an appropriate selection for VoIP services. Several cases were studied. In case of point to point and one transition node communication links, both G.723.1 and G.729 are recommended while in case of two transition nodes only G729 is the best choice. Thus, despite the fact that G.729 codec requires more bandwidth than G.723.1, the G.729 codec is better for VoIP communication via DPLC due to the shorter latency which leads to more transition nodes.

\section{Acknowledgements}

This work was supported by the Niroo Research Institute (NRI).

\section{References}

[1] Cigre, Working Group D2.23, "The use of Ethernet technology in the power utility environment", Report 460, 2011.

[2] IEC 62488-1 Ed.1, "Power line communication systems for power utility applications - Part 1: Planning of analogue and digital power line carrier systems operating over EHV/HV/MV electricity grids", IEC, 2012.

[3] Cisco, "Introduction to VoIP", Cisco publications, 2005.

[4] ITU-T Recommendation G.723.1, "Dual rate speech coder for multimedia communication transmitting at 5.3 and $6.3 \mathrm{kbps}$,, ITU-T, 2006. Online at: http://www.itu.int/rec/T-REC-G.723.1-200605-I/en.

[5] ITU-T Recommendation G.729, "Coding of speech at $8 \mathrm{kbit} / \mathrm{s}$ using CS-ACELP", ITU-T, 2012. Online at: http://www.itu.int/rec/T-REC-G.729-201206-I/en.

[6] ITU-T Recommendation G.131, "Talker echo and its control", ITU-T, 2003.2 Online at: http://www.itu.int/rec/T-REC-G.131-200311-I/en.

[7] ITU-T Recommendation G.114, "One-way transmission time", ITU-T, 2003. Online at: http://www.itu.int/rec/T-REC-G.114-200305-I/en.

[8] Cisco, "Improving and maintaining voice quality", Cisco publications, 2005. 